\title{
ISOLATED INVOLVEMENT OF CERVICAL LYMPH NODES IN CASTLEMAN'S DISEASE IN A YOUNG PATIENT: A RARE PRESENTATION
}

\author{
Madhu S. D1, Parvathi $M^{2}$, Divya Lakshmi3 ${ }^{3}$ Sneha Volvoikar 4 \\ ${ }_{1}^{1}$ Assistant Professor, Department of Radiodiagnosis, Kidwai Memorial Institute of Oncology. \\ 2 Senior Resident, Department of General Medicine, Bangalore Medical College and Research Centre. \\ 3 Post Graduate, Department of Radiodiagnosis, Kidwai Memorial Institute of Oncology. \\ ${ }^{4}$ Post Graduate, Department of Radiodiagnosis, Kidwai Memorial Institute of Oncology.
}

\section{ABSTRACT}

Isolated cervical nodal involvement of Castleman's disease is very rare, accounting for $6 \%$ of all cases. Of the two histopathological variants of Castleman's disease, the hyaline-vascular type is more common as seen in our patient and carries a better prognosis than the plasma cell type.

\section{KEYWORDS}

Cervical Lymph Nodes, Castleman's Disease, CT Scans.

HOW TO CITE THIS ARTICLE: Madhu SD, Parvathi M, Lakshmi D, et al. Isolated involvement of cervical lymph nodes in Castleman's disease in a young patient: a rare presentation. J. Evolution Med. Dent. Sci. 2016;5(39):2405-2407, DOI: $10.14260 /$ jemds/2016/559

\section{INTRODUCTION}

Castleman's disease is a rare benign lymphoproliferative disorder of unknown aetiology and pathogenesis that primarily affects lymph nodes. It can occur at any age, but is more common in 30-40 years' age group. The diagnosis of Castleman's disease is entirely based on histopathology features and on imaging the features show considerable overlap, thus posing diagnostic difficulties. The overall prognosis is good, particularly in the unicentric variety of diseases. We are hereby reporting a case of cervical nodal Castleman's disease in a 28-year-old male patient who presented with gradually progressive painless neck node swelling.

\section{CASE REPORT}

A 28-year-old male patient presented with history of multiple swellings over the neck for 2 years. Initially noticed on the right side, subsequently on the left side and progressively increasing in size over a period of 2 years. However, there was no history of fever, cough, constitutional symptoms, weight loss and loss of appetite. No history of bleeding tendency. No history of exposure to tuberculosis. No significant past medical or surgical history. On examination patient had bilateral enlarged cervical lymph nodes at all cervical nodal stations; the largest node measures $4 \times 3 \mathrm{cms}$. These nodes were non-tender, discrete, firm in consistency and mobile.

Few palpable lymph nodes noted in the left axilla, the largest measuring $2 \times 3 \mathrm{cms}$. Otherwise, his general physical examination was unremarkable. Systemic examination was normal. Provisional diagnosis of tubercular cervical lymphadenopathy and lymphoma was made.

Financial or Other, Competing Interest: None.

Submission 13-03-2016, Peer Review 29-04-2016,

Acceptance 02-05-2016, Published 16-05-2016.

Corresponding Author:

Dr. Madhu S. D

Assistant Professor

Department of Radiodiagnosis,

Kidwai Memorial Institute of Oncology,

Bangalore-29.

E-mail: sdmadhudoc@gmail.com

DOI: $10.14260 /$ jemds $/ 2016 / 559$
His investigations revealed $\mathrm{Hb}$ of $14.9 \mathrm{gm} \%$, total count of 9000 , lymphocytes $35 \%$, granulocytes $50 \%$, platelet of 3.5 lakh; peripheral smear showed normocytic normochromic blood picture with no atypical cells. His other routine investigations including RFT, LFT and RBS were normal, HIV was seronegative, Mantoux was negative. USG neck was done, which shows discrete enlarged bilateral cervical nodes with moderate vascularity on Doppler. No obvious necrosis/extracapsular spread/matted nodes noted. Chest radiograph and USG abdomen and pelvis were done and were normal. FNAC of the cervical nodes was done; the features were inconclusive and were further advised cervical nodal excisional biopsy.

In view of strong suspicion of lymphoma due to discrete nodal morphology, the patient was referred to Oncology Centre for excision biopsy and further evaluation to rule out lymphoma. On histopathology, targetoid pattern (used by pathologist) comprising of prominent follicles with vascular proliferation and hyalinisation of the germinal centres resembling Hassall's corpuscles noted. There was concentric layering of peripheral lymphocytes, interfollicular stroma showed infiltration by plasma cells, immunoblasts, eosinophils and post capillary venules. On IHC, CD23 highlighting the follicular dendritic cells, CD20 and Pax 5 positivity were seen in the follicles. These features were suggestive of Castleman's disease, hyaline vascular type. To assess the extent of nodal involvement CECT of neck, thorax, abdomen and pelvis was done, which shows homogeneous brilliantly enhancing discrete bilateral cervical nodes involving all neck nodal stations. No obvious necrosis, extracapsular spread or the matted nodes.

The rest of the neck structures including thyroid were normal. No significant nodal involvement in bilateral axilla, mediastinum, abdomen and pelvis. The rest of the thorax, abdomen and pelvis were unremarkable. 


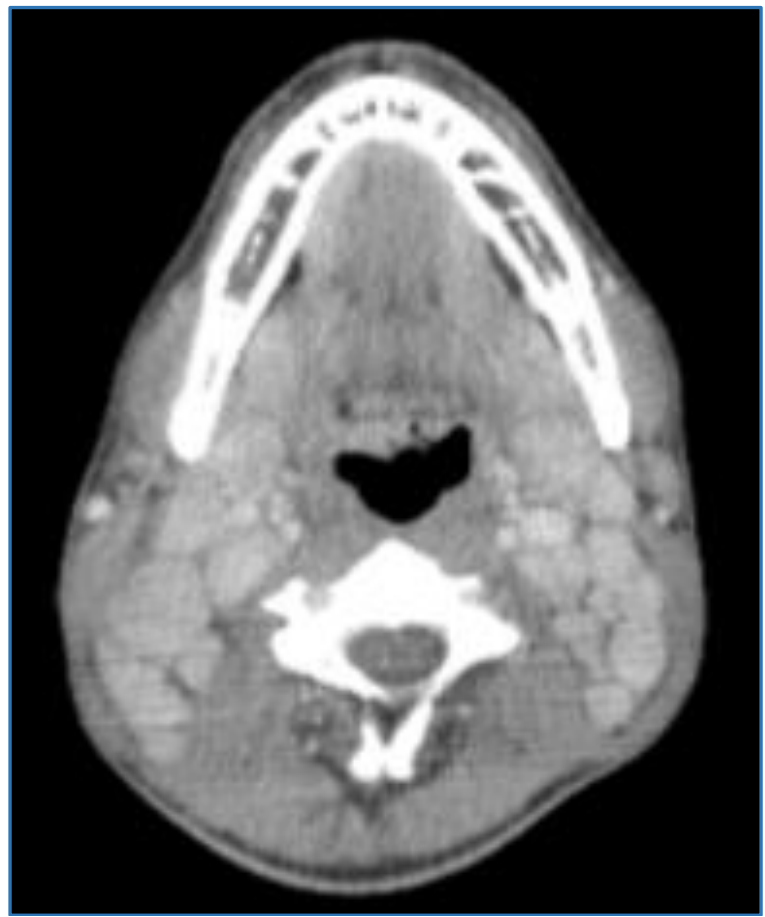

Fig. 1: Post Contrast Axial CT Scan of Neck Showing Homogeneous Brilliantly Enhancing Discrete Bilateral Level II and V Cervical Nodes with No Obvious Necrosis

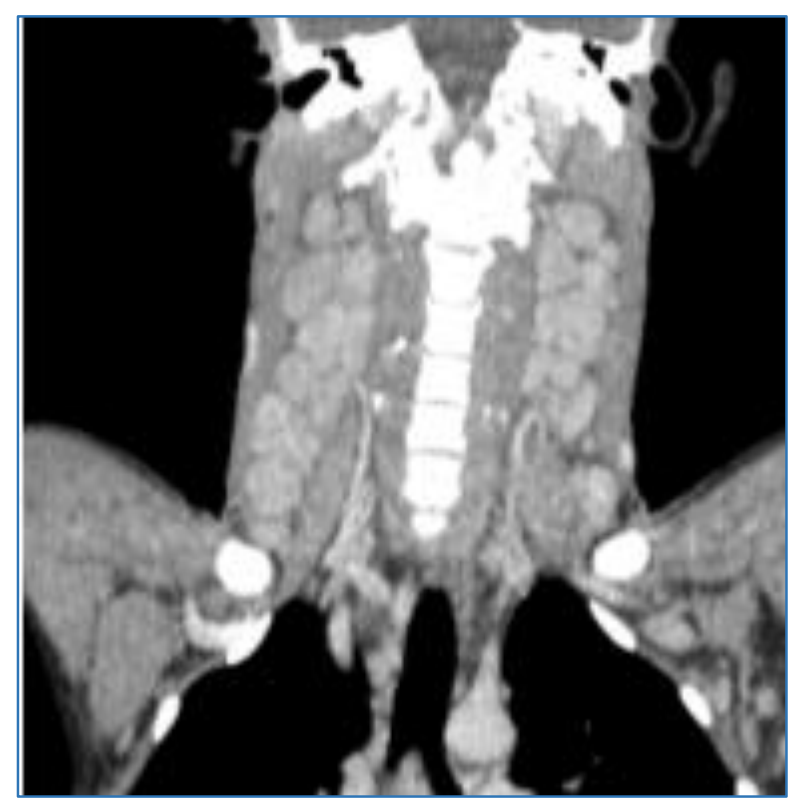

Fig. 2: Post Contrast Coronal CT Scan of Neck Showing Homogeneous Brilliantly Enhancing Discrete Bilateral Level II, III, IV and V Cervical Nodes with No Obvious Necrosis

\section{DISCUSSION}

Castleman's disease is a rare benign lymphoproliferative disorder of unknown aetiology and pathogenesis that primarily affects lymph nodes. It was first described by Dr. Benjamin Castleman in 1956 and he further reported around 13 cases of unicentric hyaline vascular Castleman's disease and described the classic pathologic features.(1) It is also known as angiofollicular or benign giant lymph node hyperplasia.(2-4) Pathologically, Castleman's disease has two major histological types as described by Keller et al in 1972; the hyaline-vascular type and the plasma-cell type. The hyaline-vascular type is far more common and accounts for $90 \%$ and is characterized by germinal follicles with hyalinised vessels surrounded by concentric layers of small lymphocytes with the proliferative interfollicular vascular stroma. The plasma cell variant is characterized by a paucity of follicular hyalinised vessels in the germinal follicles. Castleman's disease has been also classified as unicentric form or a multicentric form depending on its biologic behaviour. The unicentric form is typical of the hyalinevascular type and amenable to surgical treatment and later type typically belongs to the plasma cell type with more complicated systemic manifestations. $(3,4)$ Castleman's disease can occur at any age, but is more common in 30-40 years' age group and the multicentric form is common in the $5^{\text {th }}$ and $6^{\text {th }}$ decade.(2,4) Proposed hypotheses for the pathogenesis of Castleman's disease includes lymphoid hamartomatous hyperplasia; abnormal autoimmunity; an immunodeficiency state; but the most widely accepted theory is chronic lowgrade inflammatory process. Castleman's disease is one of the common causes of non-neoplastic lymphadenopathy and it represents a form of non-clonal lymph node hyperplasia. $(5,6)$ Around $70 \%$ of Castleman's disease cases are seen in the thorax, $10-15 \%$ in the abdomen, retroperitoneum and pelvis, and another $10-15 \%$ in the neck. This entity has also been described in the extralymphatic tissues including the lung, larynx, parotid gland, pancreas and muscle, but are very rare. $(2,4)$ The diagnosis of Castleman's disease is based on histopathology features and on imaging the features show considerable overlap, thus posing diagnostic difficulties. The overall prognosis is good, particularly in the unicentric variety of diseases.

In our case, the disease is localized to bilateral cervical nodes involving all the nodal stations with no other extracervical nodal involvement or extranodal involvement. Cervical node involvement alone is seen in approximately $6 \%$ of cases.(2,4) Clinically, this kind of presentation is also seen in tubercular adenopathy and in lymphoma in this young age group. In our case both differentials were considered, especially tubercular adenopathy as Tuberculosis is endemic in India. However, in USG neck the nodes were discrete, homogeneous without necrosis or extracapsular spread with moderate vascularity and without matted nodes. This finding made us to think of an alternate diagnosis of lymphoma. The diagnosis of Castleman's disease is confirmed by histopathology. The hyaline vascular variant typically shows hyaline vascular lymph follicles with expanded mantle zones that contain small lymphocytes forming concentric rings and an interfollicular capillary proliferation with perivascular hyalinization as in our case.

The plasma cell Castleman's disease is less distinctive at histological examination and is characterized by variable germinal centre hyperplasia and mantle zone expansion, often with relatively preserved lymph node architecture. $(2,5)$ In our case on CECT scan the cervical nodes were discrete, well-marginated showing homogeneous brilliant enhancement on contrast without necrosis or extracapsular spread. These are the typical features of Castleman's disease on CT scan. $(7,8)$ This brilliant enhancement suggests high vascularity and it is seen in hyaline-vascular variant due to its greater vascularity. On imaging three patterns of involvement have been described, which includes a solitary non-invasive mass; it is most commonly seen in $50 \%$ of cases, a dominant 
infiltrative mass with associated lymphadenopathy seen in $40 \%$ of cases and a matted lymphadenopathy without a dominant mass seen in $10 \%$ of cases. (3)

Clinical or imaging diagnosis of Castleman's disease is difficult and no definitive pre-operative diagnostic tools are available.(3) and as FNAC is usually a diagnostic and definitive diagnosis requires a biopsy. For the localized form of Castleman's disease, complete surgical resection is the mainstay of therapy with a $100 \%$ control rate for the hyaline vascular type and for patients with disseminated type; management includes a combination of radiation therapy, corticosteroids and chemotherapy.(3) This form of the disseminated verity of either histological subtype carries a poor prognosis. The plasma cell type requires excision with close follow-up and if needed a systemic chemotherapy.(9) Our case was treated with steroids and kept on follow-up with an interval decrease in size and number of nodes clinically and on USG neck. The malignant transformation of Castleman's disease is rare and its association with other malignancies like plasmacytoma, malignant lymphoma and Kaposi's sarcoma is also very rare.(10)

\section{CONCLUSION}

Castleman's disease is a rare benign lymphoproliferative disorder of unknown aetiology and pathogenesis that primarily affecting lymph nodes with very rare systemic involvement posing a diagnostic difficulty unless the patient is extensively evaluated. No definitive pre-operative diagnostic tools are available for diagnosis of Castleman's disease. Even though on CT this disease shows characteristic features, definitive diagnosis requires biopsy with histopathological assessment for confirmation.

\section{REFERENCES}

1. Castleman B, Iverson L, Menendez VP. Localized mediastinal lymphnode hyperplasia resembling thymoma. Cancer 1956;9(4):822-30.

2. Keller AR, Hochholzer L, Castleman B. Hyalinevascular and plasma-cell types of giant lymph node hyperplasia of the mediastinum and other locations. Cancer 1972;29(3):670-83.

3. McAdams HP, Rosado-de-Christenson M, Fishback NF, et al. Castleman disease of the thorax: radiologic features with clinical and histopathologic correlation. Radiology 1998;209(1):221-8.

4. Johkoh $\mathrm{T}$, Müller $\mathrm{NL}$, Ichikado $\mathrm{K}$, et al. Intrathoracic multicentric castleman disease: CT findings in 12 patients. Radiology 1998;209(2):477-81.

5. Cronin DM, Warnke RA. Castleman disease: an update on classification and the spectrum of associated lesions. Adv Anat Pathol 2009;16(4):236-46.

6. Ko SF, Hsieh MJ, $\mathrm{Ng} \mathrm{SH}$, et al. Imaging spectrum of castleman's disease. AJR Am J Roentgenol 2004;182(3):769-75.

7. Koslin DB, Berland LL, Sekar BC. Cervical castleman disease: CT study with angiographic correlation. Radiology 1986;160(1):213-4.

8. Freeman SJ, Irvine GH, Glew D. Case report: cervical castleman's disease shown by CT and MRI. Clin Radiol 1994;49(10):721-3.

9. Sanz C, Sierra J, Cobarro J, et al. An unusual case of castleman's disease restricted to the neck. ORL J Otorhinolaryngol Relat Spec 1992;54(6):331-3.

10. Case records of the Massachusetts general hospital. Weekly clinicopathological exercises (Case 32-1984). N Engl J Med 1984;311(6):388-98. 